\title{
Toward tailored medicine (and beyond): the phaeochromocytoma and paraganglioma model
}

\author{
Vincenzo Cuccurullo $\cdot$ Luigi Mansi
}

Published online: 30 May 2012

(C) Springer-Verlag 2012

The increasing availability of ${ }^{68} \mathrm{Ga}$ DOTA somatostatin analogues (SSA), at least in Europe, is challenging traditional diagnostic paths, such as that using ${ }^{123}$ I-metaiodobenzylguanidine (MIBG) in phaeochromocytomas (PCC) and paragangliomas (PGL) [1]. Here, using the PCC/PGL as the model, we discuss whether new better performing radiocompounds necessarily determine the disappearance of the older ones.

Working for the development of "tailored medicine" for each individual, and being aware that each individual decision has to be taken in the pragmatic world of cost effectiveness, the main assumptions of our thinking are:

1. It is possible that a positive result can be obtained using a radiotracer labelled with a gamma emitter but not using a radiotracer labelled with a positron emitter because of different uptake mechanisms, although using radiotracers with similar pharmacokinetics, PET is more sensitive than SPECT.

2. Comparing radiotracers with different uptake mechanisms, it is important to evaluate if positive and negative results are matched or mismatched, to identify possible advantages of a multidisciplinary approach.

3. Different radiotracers may be chosen with a preference for the best diagnosis, the best prognostic classification, or a closer connect with the therapeutic strategy. Therefore, a radiotracer with a lower sensitivity could be chosen if it more strictly corresponds to a "better" radiotherapeutic agent.

V. Cuccurullo $\cdot$ L. Mansi $(\triangle)$

Nuclear Medicine Division, Second University of Naples,

P.zza Miraglia 2,

80138 Naples, Italy

e-mail: luigi.mansi@unina2.it
4. In the cost effectiveness evaluation, the costs (including the cost of the procedure, the technology involved, the waiting list, number of hospital days, etc.) have to be calculated not for a single procedure, but with respect to the whole diagnostic and therapeutic course.

To expand the discussion, we provide information on:

1. The targets, i.e. the PCC/PGL patient groups that can be differentiated clinicopathologically.

2. The radiotracers available for clinical use.

\section{The targets}

PCC and PGL are rare neuroendocrine tumours (NET) arising in the adrenal medulla (PCC) or the extraadrenal paraganglia (PGL). The term paraganglioma includes two different tumours because, after originating from the neural crest, the paraganglia differentiate into sympathetic and parasympathetic. Sympathetic paraganglia arise from chromaffin cells along the sympathetic chains, are usually located in chest, abdomen or pelvis, and secrete catecholamine, while the parasympathetic paraganglia arise from glomera distributed along the parasympathetic nerves in the head, neck and upper mediastinum, and are nonsecreting.

The prevalence of PCC/PGL has been estimated to lie between 1:6,500 and 1:2,500 in the US. They can occur as sporadic cases, but up to $30 \%$ of patients with PGL have a family history. In $10 \%$ of patients with PCC the tumour is associated with multiple endocrine neoplasia syndrome $2 \mathrm{~A}$ or 2B, von Hippel-Lindau disease or neurofibromatosis type I. Familial PGL associated with mutations in succinate dehydrogenase complex subunit B (SDHB) shows a high rate of malignancy and hypersecretion of normetanephrine 
and norepinephrine [2]. Conversely, PGL associated with mutations in succinate dehydrogenase complex subunit D (SDHD) or $\mathrm{C}$ (SDHC) is almost always benign, and is typically located in the head and neck region. In less than $10 \%$ of patients with sporadic PCC/PGL, the tumour is extraadrenal. The majority of PCC and PGL are benign. Malignancy occurs in 5-13\% of PCC, 15-23\% of sympathetic PGL, and 2-20\% of parasympathetic PGL. The most common sites for metastases are bone, liver and lung. Currently, malignancy cannot be predicted with certainty, although some histological or genetic features might be suggestive. The prognosis of malignant PCC and PGL is poor, with a 5-year mortality rate of $>50 \%$.

While benign lesions are preferably surgically treated, therapies for malignant neoplasms may include surgical debulking, pharmacological control of hormone-mediated symptoms, radionuclide therapy, external irradiation, and systemic antineoplastic therapy. Management of recurrences involves pharmacological control of symptoms and tumour mass reduction, eventually followed by radionuclide therapy, accompanied or not by chemotherapy.

Chromogranin A, which is elevated in secretory and nonsecretory PCC/PGL, may be used to gauge tumour response and relapse because of its correlation with tumour mass [3]. Elevated levels of plasma dopamine and urinary dihydroxyphenylalanine, which occur particularly in noradrenaline-producing tumours, are more frequently associated with malignancy.

Patients affected by PCC/PGL can be divided into three groups:

1. Those with benign disease located in the adrenal glands or at the level of the paraganglia.

2. Those with malignant disease already showing metastases, but continuing to express typical markers of differentiation, such as those associated with catecholamine metabolism and/or the expression of somatostatin receptors (sst).

3. Those with a malignant "dedifferentiated" neoplasm, which has lost the typical functional and receptor characters and shows an increased and modified metabolism, mainly based on anaerobic glycolysis.

Methods to reliably predict the malignant potential of PCC/ PGL are not presently available, so diagnostic imaging may be useful for detecting tumour lesions, for defining their prognosis, for choosing the best therapeutic strategy.

\section{The radiotracers}

There are a large number of radiocompounds that have been proposed for the imaging of NET [4, 5], but not all of them have attracted interest for clinical use. Such radiocompounds include pentavalent ${ }^{99}$ Tc-dimercaptosuccinic acid (VDMSA), which is no longer commercially available, ${ }^{11} \mathrm{C}-5$ hyroxytryptophan $\left({ }^{11} \mathrm{C}\right.$-HTP) and ${ }^{11} \mathrm{C}$-hydroxyephedrine (HED), which suffer from too rapid physical decay, ${ }^{111}$ In and ${ }^{99}$ Tc SSA have the disadvantage that better results can be achieved with ${ }^{68} \mathrm{Ga}$, and ${ }^{18} \mathrm{~F}$-fluorodopamine (FDA), which is not yet widely available.

\section{Catecholamine-based imaging}

${ }^{123}$ I-Metaiodobenzylguanidine The paraganglion system comprises collections of neuroepithelial cells scattered throughout the body. In the majority of cases, PGL/PCC are differentiated tumours arising from this system with cells containing numerous catecholamine granules. MIBG is a monoamine that traces catecholamine uptake and storage, but not its metabolism. It is taken up dominantly through the neuronal uptake 1 system, and stored in the noradrenergic neurosecretory granules [6]. ${ }^{131}$ I-MIBG was developed in early 1980 s to visualize adrenal tumours. The physical characteristics and dosimetric advantages of the gamma emitter ${ }^{123}$ I support its primacy in the diagnostic field with respect to both ${ }^{131} \mathrm{I}$, which is today used only for therapeutic purposes, and the corresponding positron emitter ${ }^{124} \mathrm{I}$, which is affected by its long physical decay time.

At present, ${ }^{123} \mathrm{I}-\mathrm{MIBG}$ scintigraphy is the most diffuse radionuclide imaging modality for PCC, PGL and neuroblastoma, being essential as a prelude to ${ }^{131} \mathrm{I}-\mathrm{MIBG}$ therapy. Negative results in PCC/PGL may be due to both pathophysiological and technical causes. There is no MIBG uptake in the absence of its intracellular transporter, as may happen in the SDHB mutated form, or in the presence of dedifferentiation, i.e. in more malignant histotypes [7]. The clinical predictors for MIBG negativity are predominant norepinephrine/normetanephrine secretion, an age less than 45 years, and a hereditary cause [8]. False-negative results may also occur depending on location, being more frequent at the level of bone, or in the head and neck, or because of physical attenuation. A higher sensitivity has been observed when using ${ }^{131}$ I-MIBG at therapeutic doses, because of the count rate determined by the dosage and of the higher physical energy of ${ }^{131} \mathrm{I}$ radiation, which result in images less affected by attenuation and better detection of deep and/or bony lesions. SPECT and SPECT/CT may provide better sensitivity [9].

${ }^{18}$ F-Fluoro-L-DOPA ${ }^{18}$ F-Fluoro-L-DOPA (F-DOPA) was first proposed for use in the investigation of dopaminergic alterations, as in Parkinson's disease. F-DOPA is transported into neuroendocrine cells via the sodium-independent system L, in which a large neutral amino acid transporter (LAT1) protein linked to the glycoprotein CD98 is the principal mediator. DOPA can be decarboxylated to 
dopamine by L-amino acid decarboxylase (L-AADC), an enzyme widely expressed in differentiated NET [10]. In oncology, F-DOPA is under evaluation for the imaging of NET and neuroblastoma. Despite an uptake mechanism different from that of MIBG, leading to occasional mismatches, matched negative results have been observed in undifferentiated tumours and in some familial PCC/PGL, although a higher sensitivity of F-DOPA has been observed in SDHx-related head and neck PGL [11]. A higher sensitivity than with ${ }^{123}$ I MIBG also derives from a better spatial resolution and because of the higher energy of gamma radiation subsequent to annihilation [12]. A study comparing F-DOPA and ${ }^{131}$ I-MIBG at therapeutic dosages could provide indications for the use of F-DOPA in patients undergoing radionuclide therapy if F-DOPA is able to detect metastatic lesions negative with ${ }^{123} \mathrm{I}$ MIBG, but showing uptake after high doses of ${ }^{131}$ I MIBG.

\section{Somatostatin receptor-based imaging}

Among receptors expressed on NET cells, the most extensively studied are sst, that are G-protein-coupled receptors internalized after specific ligand binding. Starting from the SSA octreotide, a large series of molecules, all with similar pharmacokinetics, has been proposed and has attracted interest clinically both as a drug and, when radiolabelled, as a radiotracer for diagnostic and therapeutic purposes. Among the group of radiocompounds, a pivotal role is played by the first one, ${ }^{111}$ In-pentetreotide, which is still clinically useful when PET with ${ }^{68} \mathrm{Ga}$-DOTA peptides is not feasible. ${ }^{64} \mathrm{Cu}$ radiocompounds are interesting in that they allow both PET and radionuclide therapy. At present, the most diffuse therapeutic agents are DOTA peptides labelled with ${ }^{90} \mathrm{Y}$ or ${ }^{177} \mathrm{Lu}$, which are used in patients with differentiated NET, and show a favourable SSA distribution for diagnostic imaging [13].

While unfavourable results have been published for ${ }^{111}$ In- pentetreotide with respect to ${ }^{123} \mathrm{I}$ MIBG in PCC/ PGL, ${ }^{68} \mathrm{Ga}$ SSA has attracted new interest. At present, five human receptor subtypes for somatostatin have been cloned and characterized (sst1 to sst5). Variations in the binding affinities of SSA with respect to the native molecule have been demonstrated, with slight differences in diagnostic accuracy, mainly connected with their relative affinity to sst 2 and sst5, the most extensively expressed subtypes in NET. In PCC/PGL the most diffuse DOTA peptides, DOTATyr3-octreotate (DOTA-TATE), DOTA-1-Nal3-octreotide (DOTA-NOC) and DOTA-D-Phe1-Tyr3-octreotate (DOTATOC) have been proposed, and show favourable results compared to ${ }^{123}$ I-MIBG [14].

As for MIBG, SSA uptake occurs in differentiated tumours, and is lost in the undifferentiated forms. Nevertheless, a distribution different from that of MIBG (and F-DOPA) has been observed. The distribution of SSA in PCC/PGL is neither connected with catecholamine metabolism nor influenced by their derivation from sympathetic or parasympathetic paraganglia. Positive results may also be observed in familial PCC/PGL with mutations affecting catecholamine transport. Moreover, ${ }^{68} \mathrm{Ga}$ allows a higher detection of deep and bony lesions than ${ }^{123}$ I-MIBG because of a better spatial resolution and a lower negative attenuation effect.

Metabolic imaging with $\left[{ }^{18} \mathrm{~F}\right]$ fluoro-2'-deoxy-D-glucose

$\left[{ }^{18}\right.$ F]Fluoro-2'-deoxy-D-glucose (FDG) may fail to visualize well-differentiated and slow-growing tumours, such as NET. Conversely, its uptake increases in undifferentiated NET which have lost sst on cell membranes and catecholamine metabolism. This behaviour, also observed in PCC/PGL, results in a higher sensitivity than with MIBG, F-DOPA and ${ }^{68} \mathrm{Ga}$-peptides in patients with undifferentiated metastatic NET with a worse prognosis [15].

\section{Discussion and conclusion}

Both catecholamine and sst radiotracers are indicated when a benign and/or a differentiated malignant tumour is suspected, i.e. in the large majority of patients. At present, for epidemiological and practical reasons, ${ }^{123}$ I-MIBG remains the gold standard in PCC and the first-line radionuclide for the study of patients with suspected PGL. The preference for this approach in patients with PCC is justified by the high prevalence of benign disease, and is further supported by the easy location of PCC at the level of the adrenal medulla. In PGL improved sensitivity using ${ }^{123}$ I-MIBG may be obtained, mainly at the head and neck level, using SPECT and SPECT-CT. The choice of ${ }^{123}$ I-MIBG may also be favoured in metastatic differentiated PCC and PGL, when a therapeutic strategy using ${ }^{131} \mathrm{I}-\mathrm{MIBG}$ is considered.

Although the pathophysiological mechanisms of action of catecholamine and SSA both involve expression of a still partially preserved functional and cellular receptor status, they do not show complete overlap as biological targets. In particular, in some familial forms uptake of ${ }^{68} \mathrm{Ga}$-DOTA peptides and not of MIBG (and F-DOPA) has been observed. Concerning F-DOPA, although data showing a higher sensitivity than MIBG have been published, no advantages are evident with respect to ${ }^{68} \mathrm{Ga}$-DOTA peptides, also because of possible matched false-negative results between F-DOPA and MIBG in some SDHB-related PCC/PGL. Possibly the use of F-DOPA could attract new interest after a comparative study in patients undergoing radionuclide therapy with ${ }^{131} \mathrm{I}$-MIBG. 
Therefore, although a diagnostic approach based only on MIBG may be sufficient in typical PCC, in general it could be more convenient to increase diagnostic sensitivity using a multidisciplinary strategy. This premise could also be very important for therapeutic purposes if therapeutic tools using both catecholamine and somatostatin tracers radiolabelled with beta emitters with different energies and physical decay times were available.

FDG which may be positive in undifferentiated lesions and may provide better overall sensitivity in patients affected by malignant tumours, but does not have the possibility of a corresponding radionuclide therapeutic agent. Therefore, FDG may play a clinical role in prognosis or before radionuclide therapy, when a dedifferentiation is suspected, identifying patients with a worse prognosis and/or nonresponders to radionuclide therapy. A very interesting perspective is provided by the study by Martiniova et al. [16] of mouse PCC cells and tumours, demonstrating the effect of treatment with histone deacetylase inhibitors in increasing the amount of norepinephrine transporters expressed in tumours. In this way it may be possible to enhance MIBG uptake and the therapeutic efficacy of treatments with ${ }^{131} \mathrm{I}$ MIBG in patients with advanced PCC and PGL.

Therefore, in conclusion, we can substantially share the opinion of Maurice et al. [1], that ${ }^{123}$ I MIBG remains the first choice when the presence of a PCC or a PGL is suspected. ${ }^{68} \mathrm{Ga}$-DOTA peptides should be considered: (a) as the first-line investigation in patients at high risk of PGL and metastatic disease, for example for screening carriers for mutations associated with familial PGL syndromes; (b) as the next investigation if ${ }^{123}$ I-MIBG does not detect lesions in patients with a high pretest probability of PCC or PGL; and (c) in preference to ${ }^{123} \mathrm{I}-\mathrm{MIBG}$ if metastatic spread, particularly to the bone, is suspected.

This strategy, clearly supported by the contribution of CT and/or MRI, may identify a possible role in defining a malignant prognosis for FDG, while the position of FDOPA and of other radiotracers has to be further evaluated.

\section{References}

1. Maurice JB, Troke R, Win Z, Ramachandran R, Al-Nahhas A, Naji $\mathrm{M}$, et al. A comparison of the performance of (68)Ga-DOTATATE PET/CT and (123)I-MIBG SPECT in the diagnosis and follow-up of phaeochromocytoma and paraganglioma. Eur J Nucl Med Mol Imaging. 2012. doi:10.1007/s00259-012-2119-7.

2. Fliedner SM, Lehnert H, Pacak K. Metastatic paraganglioma. Semin Oncol. 2010;37(6):627-37.
3. Andersen KF, Altaf R, Krarup-Hansen A, Kromann-Andersen B, Horn T, Christensen NJ, et al. Malignant pheochromocytomas and paragangliomas - the importance of a multidisciplinary approach. Cancer Treat Rev. 2011;37(2):111-9.

4. Havekes B, Lai EW, Corssmit EP, Romijn JA, Timmers HJ, Pacak K. Detection and treatment of pheochromocytomas and paragangliomas: current standing of MIBG scintigraphy and future role of PET imaging. Q J Nucl Med Mol Imaging. 2008;52(4):419-29.

5. Ambrosini V, Tomassetti P, Franchi R, Fanti S. Imaging of NETs with PET radiopharmaceuticals. Q J Nucl Med Mol Imaging. 2010;54(1):16-23.

6. Vaidyanathan G. Meta-iodobenzylguanidine and analogues: chemistry and biology. Q J Nucl Med Mol Imaging. 2008;52(4):351-68.

7. Fonte JS, Robles JF, Chen CC, Reynolds J, Whatley M, Ling A, et al. False-negative ${ }^{123}$ I-MIBG SPECT is most commonly found in SDHB-related pheochromocytoma or paraganglioma with high frequency to develop metastatic disease. Endocr Relat Cancer. 2012;19(1):83-93.

8. Fottner C, Helisch A, Anlauf M, Rossmann H, Musholt TJ, Kreft A, et al. 6-18F-fluoro-L-dihydroxyphenylalanine positron emission tomography is superior to 123I-metaiodobenzyl-guanidine scintigraphy in the detection of extraadrenal and hereditary pheochromocytomas and paragangliomas: correlation with vesicular monoamine transporter expression. J Clin Endocrinol Metab. 2010;95(6):2800-10.

9. Fukuoka M, Taki J, Mochizuki T, Kinuya S. Comparison of diagnostic value of I-123 MIBG and high-dose I-131 MIBG scintigraphy including incremental value of SPECT/CT over planar image in patients with malignant pheochromocytoma/paraganglioma and neuroblastoma. Clin Nucl Med. 2011;36(1):1-7.

10. Minn H, Kauhanen S, Seppänen M, Nuutila P. 18F-FDOPA: a multiple-target molecule. J Nucl Med. 2009;50(12):1915-8.

11. King KS, Chen CC, Alexopoulos DK, Whatley MA, Reynolds JC, Patronas N, et al. Functional imaging of SDHx-related head and neck paragangliomas: comparison of $18 \mathrm{~F}$-fluorodihydroxyphenylalanine, 18F-fluorodopamine, 18F-fluoro-2-deoxy-D-glucose PET, 123Imetaiodobenzylguanidine scintigraphy, and 111 In-pentetreotide scintigraphy. J Clin Endocrinol Metab. 2011;96(9):2779-85.

12. Rufini V, Treglia G, Castaldi P, Perotti G, Calcagni ML, Corsello SM, et al. Comparison of 123I-MIBG SPECT-CT and 18F-DOPA PET-CT in the evaluation of patients with known or suspected recurrent paraganglioma. Nucl Med Commun. 2011;32(7):575-82.

13. Mansi L, Virgolini I. Diagnosis and therapy are walking together on radiopeptides' avenue. Eur J Nucl Med Mol Imaging. 2011;38 (4):605-12.

14. Naji M, L-Nahhas A. 68Ga-labelled peptides in the management of neuroectodermal tumours. Eur J Nucl Med Mol Imaging. 2012;39 Suppl 1:S61-7.

15. Nakazawa A, Higuchi T, Oriuchi N, Arisaka Y, Endo K. Clinical significance of 2-[18F]fluoro-2-deoxy-D-glucose positron emission tomography for the assessment of 131I-metaiodobenzylguanidine therapy in malignant phaeochromocytoma. Eur J Nucl Med Mol Imaging. 2011;38(10):1869-75.

16. Martiniova L, Perera SM, Brouwers FM, Alesci S, Abu-Asab $\mathrm{M}$, Marvelle AF, et al. Increased uptake of [123I]meta-iodobenzylguanidine, $[18 \mathrm{~F}]$ fluorodopamine, and $[3 \mathrm{H}]$ norepinephrine in mouse pheochromocytoma cells and tumors after treatment with the histone deacetylase inhibitors. Endocr Relat Cancer. 2011;18(1):143-57. 